\title{
The Effect of the Ultra-Fine Solid Additives on the Mechanical Properties of a Powder Bed ${ }^{\dagger}$
}

\author{
Makio Naito \\ Micromeritics Laboratory \\ Hosokawa Micron Corp. * \\ Jun-ichiro Tsubaki \\ Fine Ceramics Center** \\ Satoru Usuda, Naoki Kato and Genji Jimbo \\ Dept. of Chemical Engineering, \\ Nagoya University***
}

\begin{abstract}
Solid additives are widely used to control the mechanical properties of powders materials in many industries. In this paper, the measurement of the tensile strength of a powder bed with a split-cell type tester was conducted to elucidate the effect of solid additives on the mechanical properties of powders.

Fused alumina powder, silica sand, limestone, lactose and potato starch were selected as the powders. Furthermore, colloidal silica was used as a solid additive.

The relationship between the tensile strength and the porosity of a powder bed containing the additive was proposed to characterize the effect of additives on the mechanical properties of the powders.

Furthermore, the experimental results reported by others were able to be explained by the above relationship.
\end{abstract}

\section{Introduction}

Solid additives are widely used in industries as glidants to improve flowability, as lubricants, and as grinding aids, among others. These additives control the mechanical properties of powders. Furthermore, the use of solid additives is considered to be a convenient method for future treatment in the design of various powders. However, an established theory has not yet been developed for the

* No. 9, Soudai Tajika 1-chome, Hirakata, Osaka, 573 TEL. 0720 (55) 2220

** 2-4-1, Rokuno, Atsuta-ku, Nagoya, 456 TEL. 052 (871) 3500

*** Furo-cho, Chikusa-ku, Nagoya, 464 TEL. 052 (781) 5111

$\dagger$ This report was originally printed in J. Soc. Powder Technology, Japan, 24, 455-461 (1987) in Japanese, before being translated into English with the permission of the editorial committee of the Soc. Powder Technology, Japan. mechanism of their action ${ }^{5)}$. Systematic studies have not yet been undertaken on the effect of solid additives on the tensile strength (one basic property) of a powder bed. Thus, there is a lack of experimental studies on the manner in which solid additives act, even though there are a few relevant reports $1,3,8$ ).

In this regard, systematic studies were conducted by the authors ${ }^{6,7)}$ on the effect of an additive on the tensile strength of a powder bed, starting with colloidal silica (finely divided anhydrous silica), which is in general use as an additive. The results showed ${ }^{6)}$ that if the additive content is used as a parameter, the tensile strength of a powder bed containing an additive can be represented by the experimental equation $^{4)}$

$$
\sigma_{z}=k_{1 n} \exp \left(-\epsilon / b_{n}\right)
$$

which has already been proposed. However, these studies did not elucidate the manner in which the additive acted because the coeffi- 
cients $k_{1 n}$ and $b_{n}$ in Eq. (1) varied as the additive content changed. They also varied as the powder was changed in kind and in particle $\operatorname{size}^{6)}$

In this paper, a new characterization method is proposed to evaluate data of the tensile test of a powder bed containing an additive. Furthermore, this characterization methods is applied to the evaluation of the effect of ultra-fine solid additives on other mechanical properties.

\section{Experiments}

\section{1 Method and apparatus used in the ex- periments}

The experiments consist of the mixing of a powder with an additive and of the tensile test of the mixed powder. The test specimen was mixed in a V-type mixer containing 20 rubber balls, each with a diameter of $30 \mathrm{~mm}$ (VJS, product of Tsutsui Physicochemical Instruments Co.). Prior to the mixing of the specimen powder, the material and the additive were made to stand in a thermohygrostat vessel under a specified constant humidity for 24 hours. Then, a batch of the two weighing a total of $0.5 \mathrm{~kg}$ was mixed in a mixer at 50 r.p.m. for 2 hours. The resultant mixture was again conditioned in the thermohygrostat vessel and then subjected to the tensile test. A specimen powder containing no additive was also prepared by mixing this way, and the resultant powder was subjected to the test itself.

A split cell type tester (Cohetester, product of Hosokawa Micron Corp.), shown in Fig. 1, was employed in the tensile test of the powder beds. The cell had an inside diameter of $50 \mathrm{~mm}$ and a depth of $20 \mathrm{~mm}$. The test was carried out

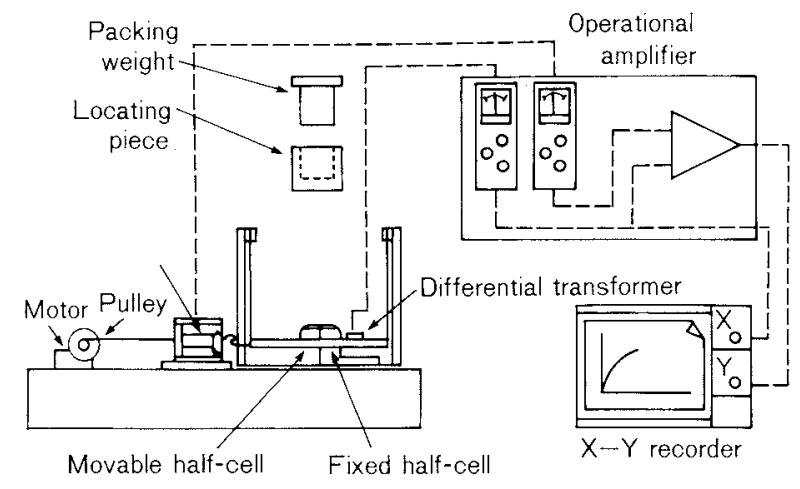

Fig. 1 Split cell type tensile strength tester by a procedure in which, first, the cell was filled with a specimen powder using a spatula. The specimen was then placed under pressure for 10 minutes for pre-compression to adjust the porosity of the powder bed. Next, the top of the powder bed in the cell was leveled so that the powder bed assumed a set volume, and then the tensile strength was measured by pulling the movable half-cell. The applied precompressive stress was within the range of $1.55 \sim 11.5 \mathrm{kPa}$, and the porosity of the powder bed was measured by weighing the specimen after the tensile test. The apparent particle density $\rho_{n}$ of the powder containing the additive was calculated by the following equation,

$$
\rho_{n}=\frac{1}{(1-n) / \rho_{p}+n / \rho_{a}}
$$

Since additives are generally used in minute ratios, the additive content in the present experiments ranged up to a maximum weight fraction of 0.04 . The tensile test was carried out twice for each combination of the same values of the additive content and the precompressive stress. The mean values of the tensile strength and of the porosity thus obtained were used in the subsequent analysis. Both the thermohygrostat vessel and the environment in which the tensile test was carried out were conditioned at a constant temperature of $20^{\circ} \mathrm{C}$, and a constant relative humidity of $50 \%$.

\section{2 The specimen powders}

Table 1 shows the abbreviations used in Figs. $2 \sim 5$ as well as the mean diameter and the true density of the particles of each of the specimen powders and of the additive used. Both the lactose and potato starch conformed to Japanese Pharmacopoeia. The particle size of each specimen powder was measured by a laser diffraction method (CILAS), and the $50 \%$ diameter obtained by integration of the volumes was used as the mean particle diameter. Figure 2 shows the particle size distributions of the specimen powders. Solid additives were available in a variety of types, but as in the previous papers ${ }^{6,7)}$, a colloidal silica (AEROSIL 130, product of Nihon Aerosil Co.), which is in wide use as an additive to improve 
Table 1 Powder materials and additive

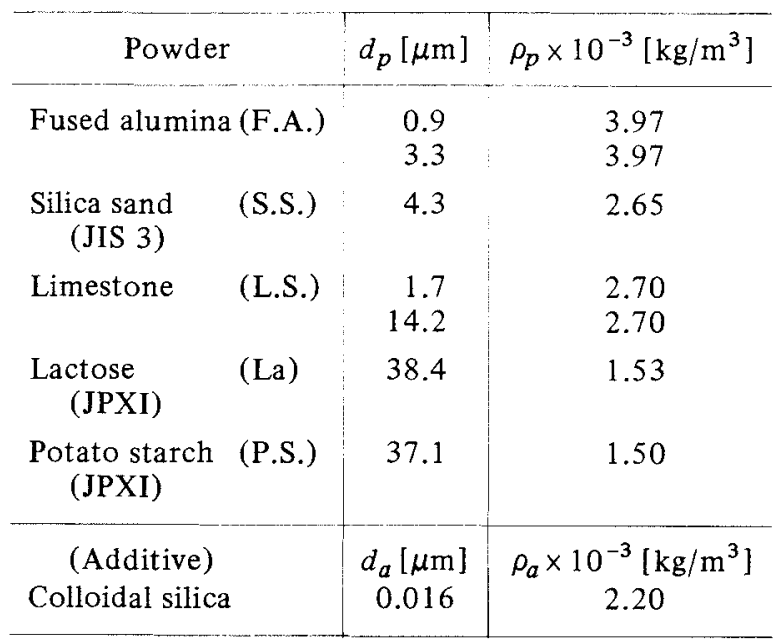

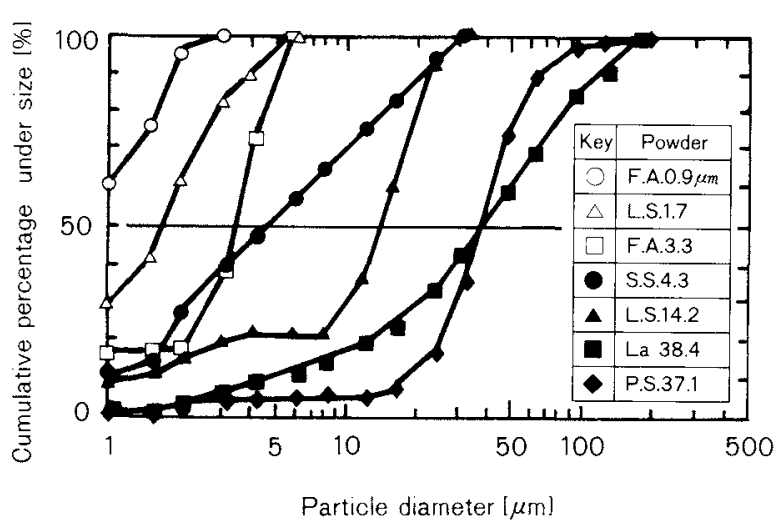

Fig. 2 Particle size distributions of powders

flowability, was used as the solid additive. The mean particle diameter of the additive was the value shown in the catalog.

\section{The results of the experiment}

Because the data was processed with the additive content $n$ as a parameter in the previous paper $^{6)}$, the pre-compressive stress $p$ was used as a parameter in this paper. Furthermore, the data was processed into relationships of the additive content $n$ with the tensile strength $\sigma_{z}$ and porosity $\epsilon$, respectively. Figures 3 and 4 show examples of data obtained for a precompressive stress $p$ of $6.54 \mathrm{kPa}$.

With respect to the relationship between the tensile strength $\sigma_{z}$ of powder bed and the additive content $n$, it is seen from Fig. 3 that the tensile strength $\sigma_{z}$ of each powder bed decreased as the additive content $n$ increased. Furthermore, the curves indicate that for additive contents $n$ at the initial stage of the ad-

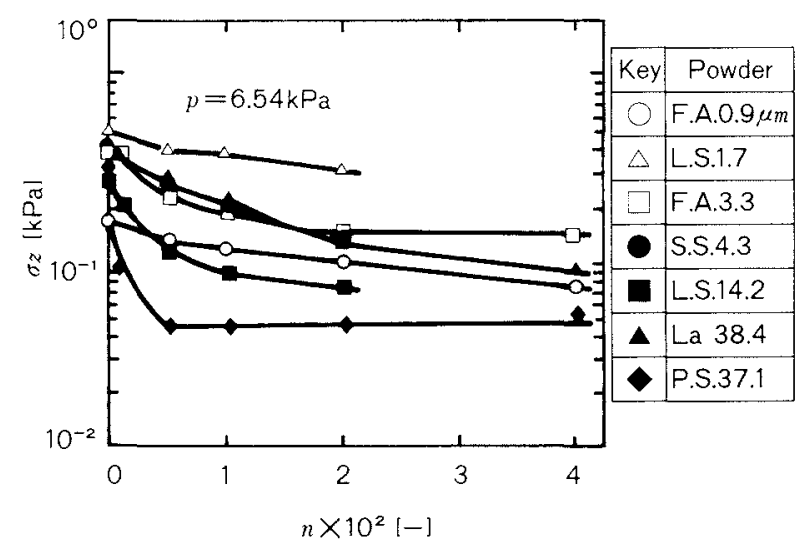

Fig. 3 Relationship between tensile strength of powder bed and additive content

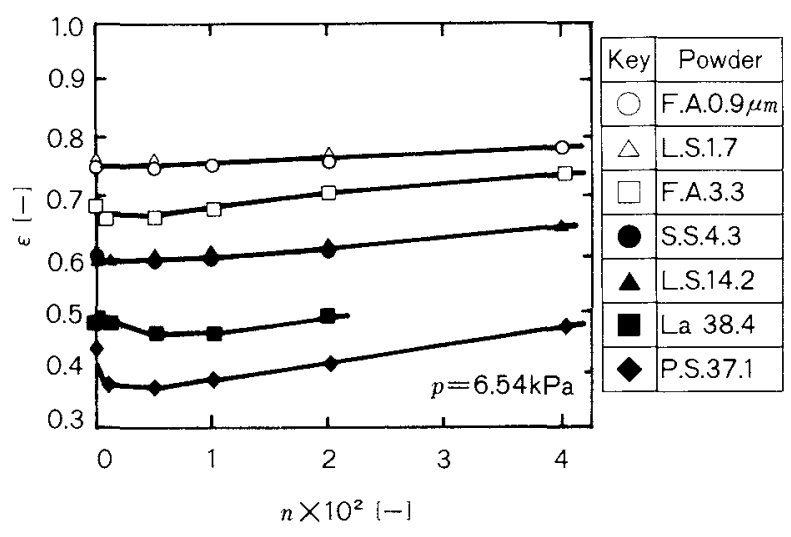

Fig. 4 Relationship between porosity of powder bed and additive content

dition, larger decreases in the tensile strength occurred where the particle diameter of the powder was larger. It was also observed by electron microscopic photography that ultrafine particles of additive were scattered on the surface of the powder particle and attached to ${ }^{i t^{6}}$. Therefore, on the presumption that the rate of change in the tensile strength is correlative to the number of the particles of additive per unit of powder surface area, a conversion of the additive content $n$ into the number of the particles of additive per unit of powder surface area was done by Eq. (3),

$$
N=\frac{n / \frac{\pi}{6} \rho_{a} d_{a}^{3}}{(1-n) \times 6 / \rho_{p} d_{p}}
$$

The calculation gave no regard to the particle size distributions of the additive or of the powder but was based on the mean particle diameters $d_{p}$ and $d_{a}$. Furthermore, the tensile strength $\sigma_{z}$ was normalized as follows so as to 


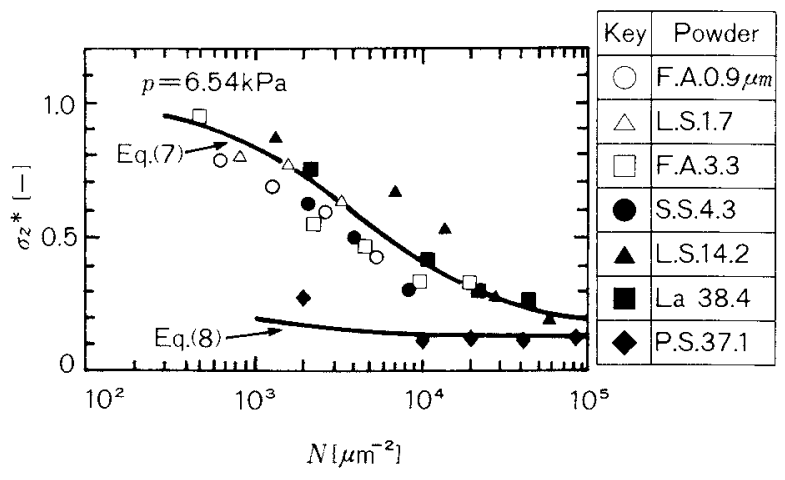

Fig. 5 Relationship between $\sigma_{z}{ }^{*}$ and number of additives per unit powder surface area

simplify the comparison of the tendencies of its changes:

$$
\sigma_{z}^{*}=\sigma_{z} / \sigma_{z n=0}
$$

Using the above process, the relationship in Fig. 3 is reduced to the relationship between $\sigma_{z}{ }^{*}$ and $N$ in Fig. 5. With the exception that $\sigma_{z}{ }^{*}$ is distinctly lower in potato starch than in the other powders, it is seen that, although the data shows some scatter, $\sigma_{z}^{*}$ can be reduced to a one single curve. This curve indicates that $\sigma_{z}{ }^{*}=1$ for $N=0$, and it shows that the decrease in $\sigma_{z}^{*}$ gradually develops a tendency to level off as $N$ is increased. An approximation of this curve was done by the equation,

$$
1-\sigma_{z}^{*}=\frac{a b N}{1+a N}
$$

To examine the relationship between Eq. (5) and the experimental data, Eq. (5) was transformed into Eq. (6) so that the relationship between $N /\left(1-\sigma_{z}^{*}\right)$ and $N$ was plotted in a diagram in Fig. 6.

$$
\frac{N}{1-\sigma_{z}^{*}}=\frac{1}{a b}+\frac{N}{b}
$$

From Fig. 6 it is seen that, independent of the pre-compressive stress and except for potato starch, the data of the powders, can be reduced to one straight line. Although the coefficients $a$ and $b$ are different, the data with respect to potato starch can also be represented by Eq. (5). Accordingly, an experimental equation,

$$
\begin{aligned}
\sigma_{z}^{*}= & 0.18+\frac{0.824}{1+\left(2.63 \times 10^{-4}\right) N} \\
& \left(a=2.63 \times 10^{-4} \mu \mathrm{m}^{2}, b=0.824\right)
\end{aligned}
$$

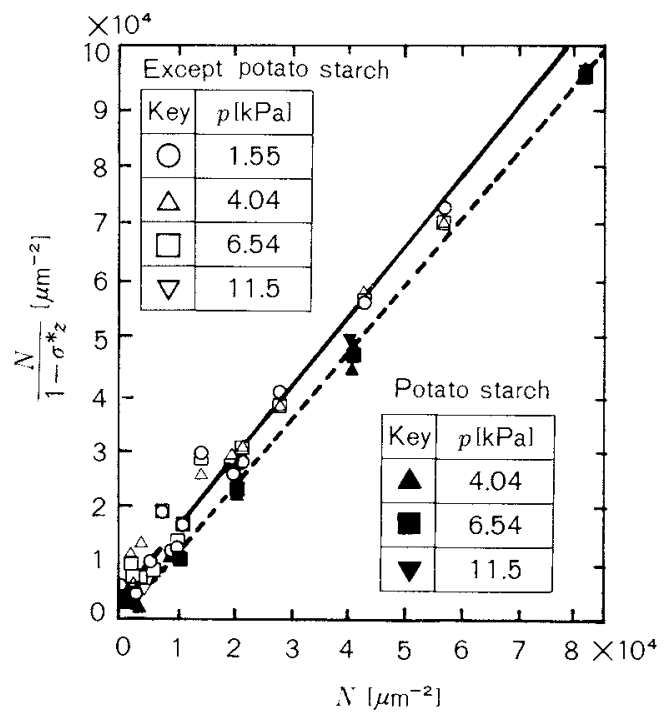

Fig. 6 Evaluation of experimental constants $a$ and $b$ of Eq. (5)

can be proposed for powders other than potato starch. Also, an experimental equation,

$$
\begin{aligned}
\sigma_{z}^{*}= & 0.14+\frac{0.859}{1+\left(1.53 \times 10^{-2}\right) N} \\
& \left(a=1.53 \times 10^{-2} \mu \mathrm{m}^{2}, b=0.859\right)
\end{aligned}
$$

can be proposed for potato starch. The coefficients $a$ and $b$ were determined by the leastsquares method from the data in Fig. 6. Figure 5 shows the results of the calculations using the above experimental equations. It was ascertained that Eqs. (7) and (8) were in fair agreement with the experimental data. From the above processing, it has become clear that the changes in the tensile strength that occur when an ultra-fine powder additive is used can be experimentally represented by Eq. (5).

A study of the relationship between the porosity $\epsilon$ of the powder bed and the additive content $n$ in five types of specimen powders has made it clear that, as shown in Fig. 4, there is a distinct difference between the inorganic powders (fused alumina, silica sand, and limestone) and the organic powders (lactose and potato starch). With respect to the inorganic powders, the porosity scarcely changes when the additive content is under 0.01 , but the porosity of each powder tends to increase as the additive is increased to a content above 0.01 . On the other hand, the porosity of each of the organic powders decreases to a mini- 
mum at a certain additive content. To make the comparison of this data easier, the porosities were normalized in the same manner as Eq. (4), by Eq. (9),

$$
\epsilon^{*}=\epsilon / \epsilon_{n=0}
$$

Figure 7 shows the resultant data into which data obtained for different pre-compressive stresses has also been incorporated. With respect to the values of $\epsilon^{*}$ of the inorganic powders for each additive content, their respective mean values have been plotted. The ranges of the data are shown by the standard deviation $\sigma$ in Fig. 7. Concerned with the inorganic powders, it can be seen from Fig. 7 that $\epsilon^{*}$ can be represented by the additive content $n$ rather than by the pre-compressive stress.

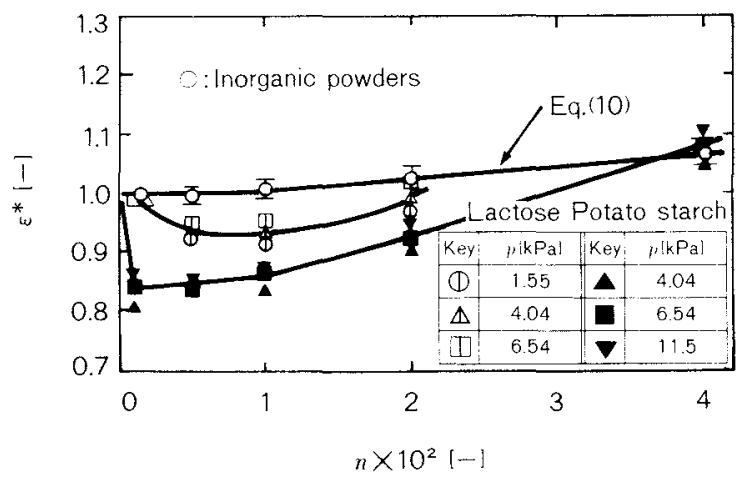

Fig. 7 Relationship between $\epsilon^{*}$ and additive content
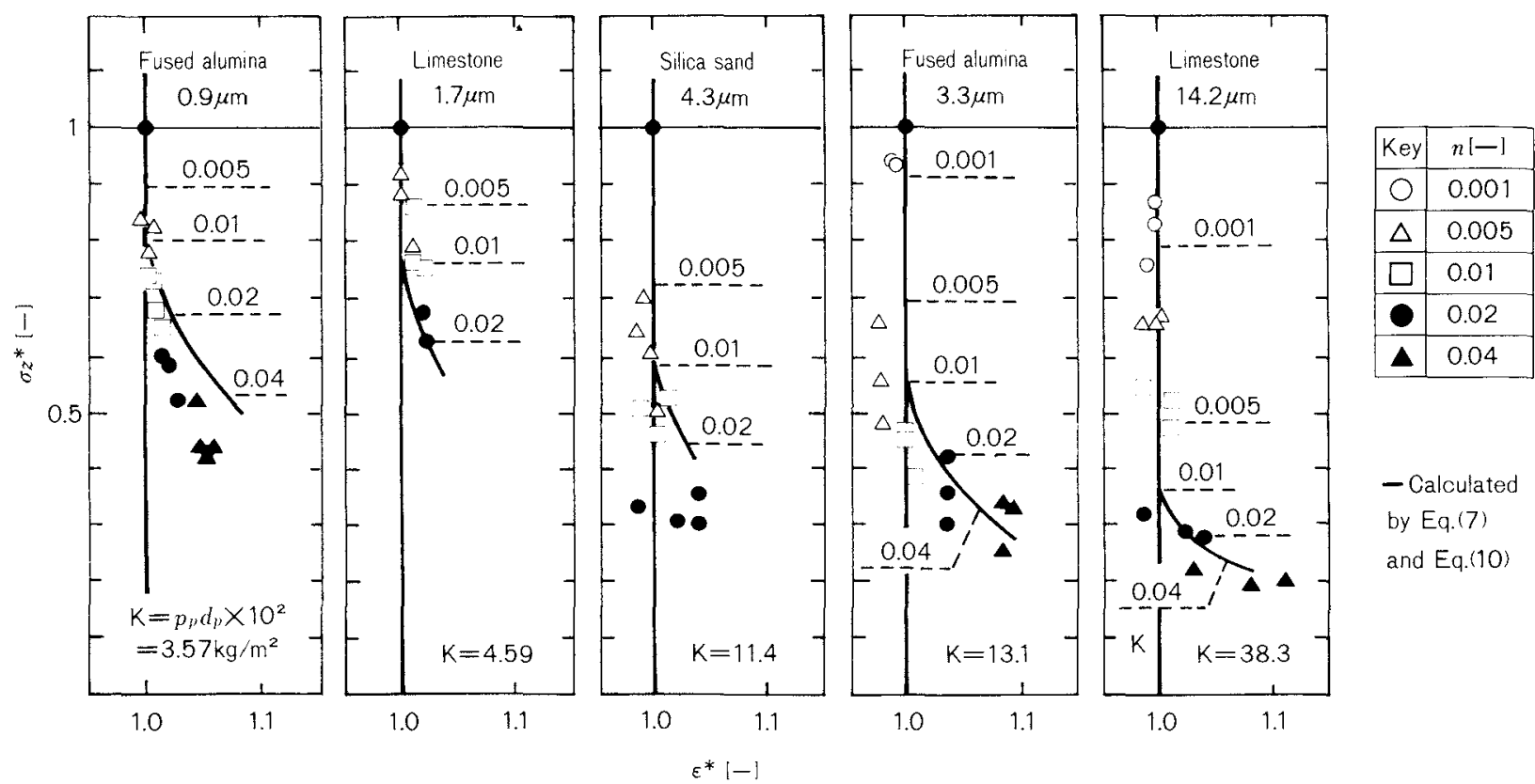

This Eq. (10) plotted in Fig. 7 agrees well with the experimental data.

The relationships of the additive content $n$ with the tensile strength $\sigma_{z}^{*}$ and the porosity $\epsilon^{*}$ of the powder bed have been reviewed, respectively. Next a study of the relationships between $\sigma_{z}^{*}$ and $\epsilon^{*}$, shown in Figs. $8 \sim 10$, is done. In these figures, the solid lines represent the calculation of the results obtained by Eqs. (3), (7), (8), and (10), while $\epsilon^{*}$ of the organic powders represents the mean values of $\epsilon^{*}$ at each additive content shown in Fig. 7. The calculated values of $\left(\epsilon^{*}, \sigma_{z}^{*}\right)$ for each additive content are also shown in Figs. $8 \sim 10$. From Fig. $8 \sim 10$, it is seen that, although there are small differences between the data and the experimental equations, they are in close agreement with respect to the representation of the changes in the tendencies of $\sigma_{z}^{*}$ and $\epsilon^{*}$. This

Fig. 8 Relationship between $\sigma_{z}^{*}$ and $\epsilon^{*}$ (Inorganic powders) (I: $n \leqq 0.01, \mathrm{I}: 0.01 \leqq_{n}$ ) 


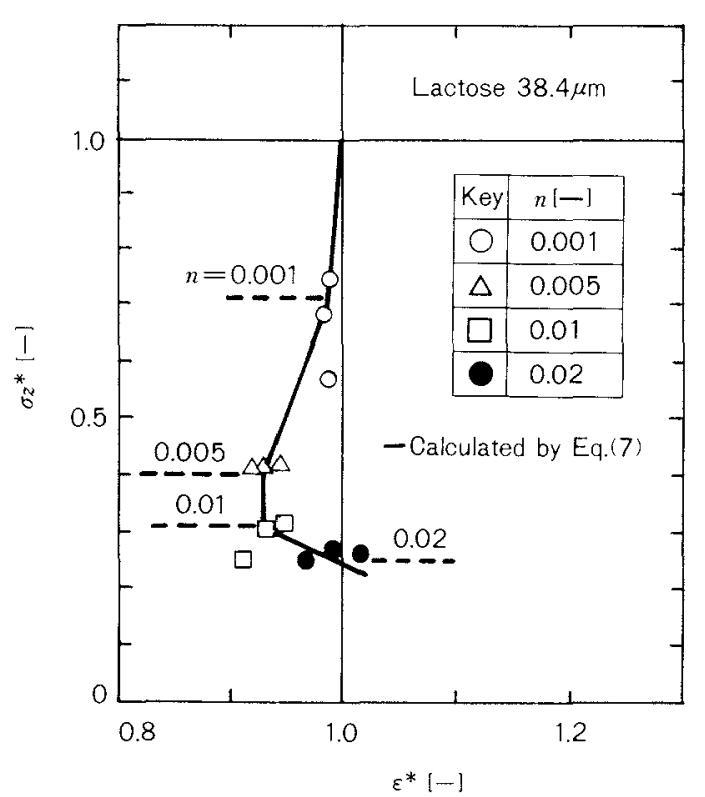

Fig. 9 Relationship between $\sigma_{z}{ }^{*}$ and $\epsilon^{*}$ (lactose) (I: $n \leqq 0.005$, II : $0.005 \leqq_{n} \leqq 0.01$, III : $0.01 \leqq_{n}$ )

proves that the foregoing experimental equations are applicable to the evaluation of the data in Figs. $8 \sim 10$.

\section{Discussion}

First, consider the scattering of $\sigma_{z}^{*}$ and $\epsilon^{*}$ in Figs. $8 \sim 10$. In reviewing the data in Figs. $8 \sim$ 10 , the influence of the additive content $n$, disregarded the pre-compressive stress $p$, has been considered so far. Now, reconsider the effect of the pre-compressive stress $p$ on $\sigma_{z}^{*}$ and $\epsilon^{*}$. Figure 11 shows a portion of the results from the reconsideration of Figs. 8 10. From Fig. 11, it is seen that where the applied pre-compressive stress is relatively large, limestone $14.2 \mu \mathrm{m}$ and lactose show only a slightly larger increase in $\epsilon^{*}$ as the additive content is increased. On the other hand, fused alumina powder shows that $\sigma_{z}{ }^{*}$ is influenced by the pre-compressive stress. Much of the scatter that the data shows when it is processed by the additive contents only, as shown in Figs. $8 \sim 10$, is due to the influence of the pre-compressive stress $p$, which is large enough not to be entirely disregarded, as shown in Fig. 11. The above-mentioned influence of the pre-compressive stress is a matter of interest when the manner in which additives act is studied. This remains to be studied further in the future. However, we will proceed

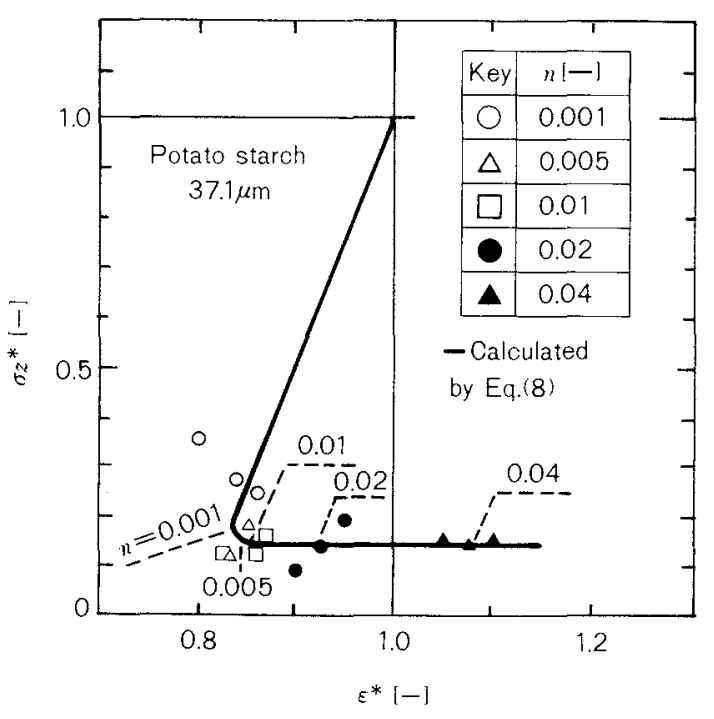

Fig. 10 Relationship between $\sigma_{z}^{*}$ and

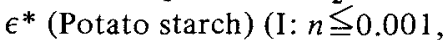
II : $0.001 \leqq n \leqq 0.01$, III : $0.01 \leqq n$ )

with our studies in this paper without considering the influence of the pre-compressive stress, because of the limited range in which the pre-compressive stress was used in the experiments. Unfortunately, experimental data and data for ascertaining accuracy are lacking in Fig. 11.

As mentioned in the introduction, papers dealing with the effect of additives on tensile strength are extremely limited. Only the results of Hayashi et al. ${ }^{1)}$ relating to potato starch are adequate. Processing their data ${ }^{1)}$ in the same manner as was done for Figs. $8 \sim 10$, in Fig. 12 is obtained. The experiments were carried out at a constant pre-compressive stress $p$ of 9.99 $\mathrm{kPa}$. This was done using colloidal silica as the additive $\left(d_{a}=0.012 \mu \mathrm{m}\right.$, AEROSIL 200, product of Nihon Aerosil Co.), which differs in particle diameter from the additive in this paper. In Fig. 12, the solid line represents an experimental equation in which the data was processed using Eq. (5). The experimental equation was,

$$
\begin{aligned}
\sigma_{z}^{*}= & 0.13+\frac{0.869}{1+\left(1.05 \times 10^{-3}\right) N} \\
& \left(a=1.05 \times 10^{-3} \mu \mathrm{m}^{2}, b=0.869\right)
\end{aligned}
$$

Measured values were used to represent $\epsilon^{*}$ in Fig. 12. The close agreement between the calculated and measured values in this figure suggests the general applicability of the present process. 


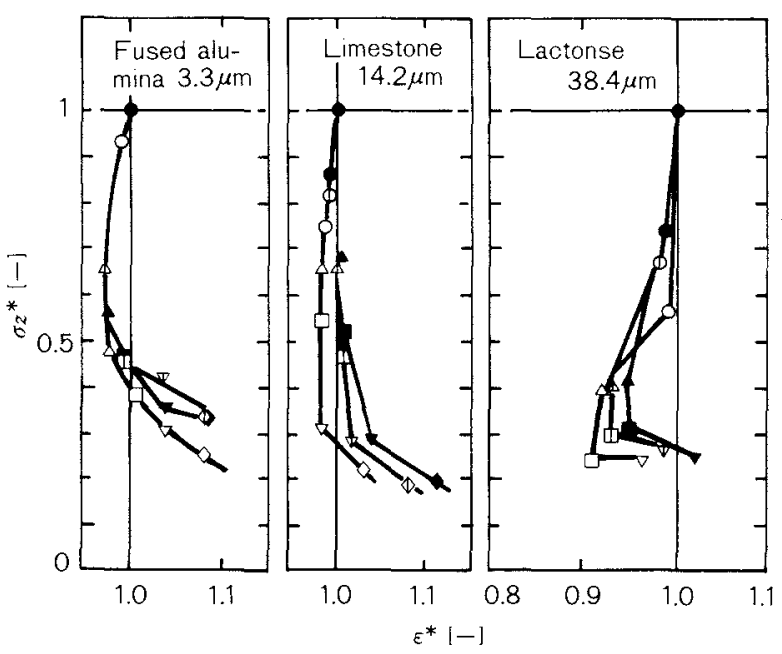

\begin{tabular}{|c|c|c|c|}
\hline$n$ & $1.55 \mathrm{kPa}$ & $4.04 \mathrm{kPa}$ & $6.54 \mathrm{kPa}$ \\
\hline 0.001 & $\bigcirc$ & $\Phi$ & $\bullet$ \\
\hline 0.005 & $\Delta$ & $\Delta$ & $\boldsymbol{\Delta}$ \\
\hline 0.01 & $\square$ & $\square$ & $\square$ \\
\hline 0.02 & $\nabla$ & $\nabla$ & $\nabla$ \\
\hline 0.04 & $\diamond$ & $\Phi$ & \\
\hline
\end{tabular}

Fig. 11 Effect of pre-compressive stress on relationship between $\sigma_{z}^{*}$ and $\epsilon^{*}$

The manner in which ultra-fine powder additives act will now be studied by applying the process used in Figs. $8 \sim 10$ and Fig. 12. Since no relevant data is available on inorganic powders, except that in this paper, our study is based on the data obtained in this paper. As shown in Fig. 8, the data can be divided into two steps by the additive content as follows:

$$
\begin{array}{lll}
\text { Step I } & (n \leqq 0.01): \epsilon^{*}=1, & \sigma_{z}^{*} \downarrow \\
\text { Step II } & (n \geqq 0.01): \epsilon^{*}, & \sigma_{z}^{*} \downarrow
\end{array}
$$

From a comparison of the differences between different powder materials, it is found that $\sigma_{z}^{*}$ at an additive content $n$ of 0.01 tends to be smaller when $K\left(=\rho_{p} d_{p} \times 10^{2}\right)$ is larger. In other words, the larger $\rho_{p} \cdot d_{p}$ is, the larger the range in which $\sigma_{z}{ }^{*}$ is, assuming Step I.

With respect to organic powder materials, the data, into which the results of Hayashi et al. has been incorporated, can be divided into the following three steps:

$$
\begin{aligned}
& \text { Step I } \quad \epsilon^{*} \downarrow \quad, \sigma_{z}^{*} \downarrow \\
& \text { Step II } \epsilon^{*}=\text { const, } \sigma_{z}^{*} \downarrow \\
& \text { Step III } \quad \epsilon^{*} \uparrow \quad, \sigma_{z}^{*} \downarrow
\end{aligned}
$$

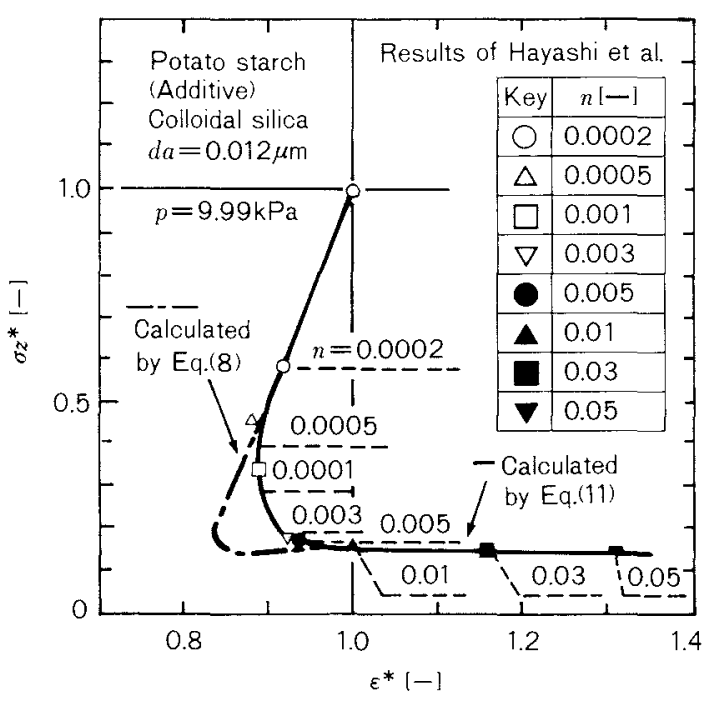

Fig. 12 Relationship between $\sigma_{z}{ }^{*}$ and $\epsilon^{*}$ of experimental results of Hayashi et al. ${ }^{1)}$

The data of the lactose shown in Fig. 9 obtained at an additive content $n$ under 0.005 comes under Step I, that obtained at $n$ within the range of $0.005 \sim 0.01$ comes under Step II, and that obtained at $n$ above 0.01 comes under Step III. As shown in Fig. 10, the data on potato starch obtained by the authors comes under Step I for an additive content $n$ under 0.001 , under Step II for $n$ within the range of $0.001 \sim 0.01$, and under Step III for $n$ above 0.01 . The data obtained by Hayashi et al., as shown in Fig. 12, comes under Step I for an additive content $n$ under 0.0005 , under Step II for $n$ within the range of $0.0005 \sim 0.003$, and under Step III for $n$ above 0.003 .

The characteristics that the organic powders exhibit in each step are as follows: In Step I, as shown by the decrease of $\sigma_{z} *$ more rapidly than of $\epsilon^{*}$, the additive is seen to have a dominant influence upon the decrease in tensile strength of the powder bed. On the other hand, in Step III, as shown by the increase of $\epsilon^{*}$, which occurs at a conspiciously higher rate in the case of potato starch than the decrease in $\sigma_{z}{ }^{*}$, the additive is seen to act dominantly toward the increase in the porosity of the powder bed. Step II is a transition region from Step I to III, where $\epsilon^{*}$ changes only slightly while $\sigma_{z} *$ decreases.

We have now characterized the manner in which an additive acts on the tensile test data 


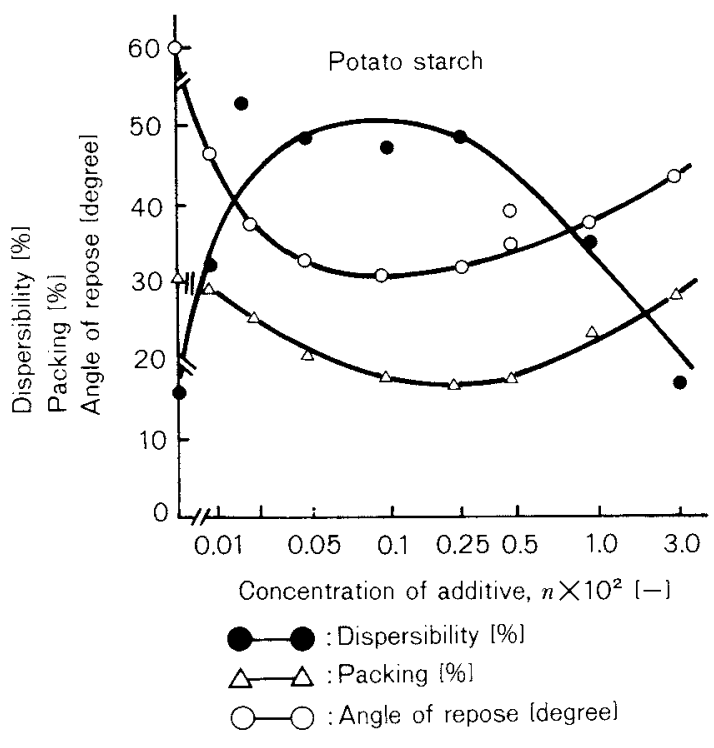

Fig. 13 Effect of additive on mechanical properties of potato starch powder reported by Hayashi et al. ${ }^{1}{ }^{\text {) }}$

of both inorganic and organic powders by the processing method using the data in Figs. $8 \sim$ 10 and Fig. 12. Next, consider the interrelations between the characteristics that have been observed and the changes in the other mechanical properties that are caused by additives.

Besides the tensile test shown in Fig. 12, the studies of Hayashi et al. ${ }^{1)}$ were extended to the effect of an additive on the dispersibility, compressibility by tapping, and angle of repose. The results are shown in Fig. 13. In their paper, they state that on the basis of the tensile test data, they calculated the adhesive force for one particle using Rumpf's equation ${ }^{9)}$ so that they were able to minimize the adhesion force when the concentration of the additive was approximately 0.003 . Their paper ${ }^{1)}$ states that the adhesion force increases before and after the above-mentioned concentration, and that these changes in the adhesion formed a pattern similar to those of the changes in the mechanical properties of the powder material, as shown in Fig. 13.

The changes in the mechanical properties of powder caused by an additive, shown in Fig. 13, are considered to be composed of three steps: In one step, $(n \leqq 0.0005)$ the dispersibility increases, and both the compressibility by tapping and angle of repose decrease

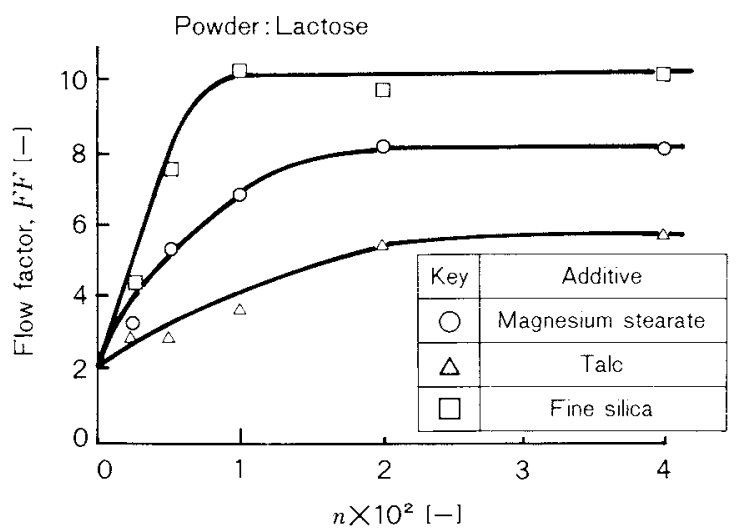

Fig. 14 Relationship between Flow Factor (FF) and additive content reported by P. York ${ }^{10)}$

with the concentration of the additive; next, virtually no change is observed in each of the three properties $(0.0005 \leqq n \leqq 0.003)$; and in the third step, $(0.003 \leqq n)$ the dispersibility decreases, and both the compressibility by tapping and angle of repose increase. Therefore, it is considered impractical to explain the relationships shown in Fig. 13 of this paper on the basis of the observations by Hayashi et al.

However, application of the processing method that has been proposed by the authors brings out the correlation of the three steps in Fig. 13 to the three shown in Fig. 12, and it makes it possible to evaluate the changes in dispersibility, compressibility by tapping, and angle of repose in Fig. 13 on the basis of Fig. 12.

P. York ${ }^{10}$ ) has reported on his studies regarding the relationship between the flow factor (FF) and the additive content, which was proposed by A.W. Jenike ${ }^{2)}$. Figure 14 shows the results reported by York for which the lactose was used. From this figure, it is seen that FF increases with the addition of the additive until the additive content reaches 0.01 , and then develops a tendency in which the additive contents level off above 0.01 . In Fig. 9, on the other hand, the additive content of 0.01 corresponds to a transition point of an organic powder from Step II to Step III in the observations by the authors. This correspondence also suggests the applicability of the characterization method presented in this paper to the evaluation of changes in FF. From the above-mentioned observations, the characteri- 
zation of the manner in which additives act, which this paper deals with using Figs. $8 \sim 10$ and Fig. 12, is also considered to be important in the evaluation of changes in other mechanical properties caused by additives.

\section{Conclusion}

Our observations on tensile tests, to which powder beds containing colloidal silica as an additive were subjected, are summarized as follows:

1) The changes in the tensile strength of a powder bed containing an additive are experimentally processed by the number of the particles of the additive per unit of powder surface area, and the changes in the porosity of the powder bed are processed by the additive content based on mass.

2) The relationship between the tensile strength and the porosity of a power bed containing an additive, for which 5 kinds of powders were subjected to the experiments in this paper, is characterized in two steps for inorganic powders (fused alumina powder, silica sand, and limestone) and in three steps for organic powders (lactose and potato starch).

3) The above-mentioned characterization has also been found to be important in evaluating the effect of an additive on other mechanical properties of powders.

\section{Nomenclature}

$a$ : experimental constant of Eq. (5)

$b$ : experimental constant of Eq. (5)

$\left[\mu \mathrm{m}^{2}\right]$

$b_{n}$ : experimental constant of Eq. (1)

$d_{a}$ : mean particle diameter of additive

$[\mu \mathrm{m}]$ $d_{p}$ : mean particle diameter of powder

$K: \rho_{p} \cdot d_{p} \times 10^{2}$

$[\mu \mathrm{m}]$

$k_{1 n}$ : experimental constant of Eq. (1)

$N$ : number of additives per unit powder surface are

$n$ : additive weight content

$p$ : pre-compressive stress

$\left[\mu \mathrm{m}^{-2}\right]$

$\epsilon$ : porosity of powder bed

$\epsilon$ : porosity of powder bed

$\epsilon_{n=0}$ : porosity of powder bed at $n=0$

$\epsilon^{*}: \epsilon / \epsilon_{n}=0$

$\rho_{a}$ : true additive density

$\rho_{n}$ : apparent particle density

$\rho_{p}:$ true particle density

$\sigma_{z}$ : tensile strength of powder bed

$\left[\mathrm{kg} / \mathrm{m}^{3}\right]$

$\sigma_{z n=0}:$ tensile strength of powder bed at $n=0 \quad[\mathrm{kPa}]$

$\sigma_{z}^{*}: \sigma_{z} / \sigma_{z n=0}$

\section{References}

1) Hayashi, H., T. Kasano and K. Suhara: Yakuzaigaku, 39, 187 (1979).

2) Jenike, A.W.: Bull. of the Univ. of Utah, No. 108 (1961).

3) Jimbo, G., S. Asakawa, N. Soga, S. Hatano and T. Uchiyama: J. Soc. Materials Sci, Japan, 18, 541 (1969).

4) Jimbo, G. and R. Yamazaki: Preprints of European Symposium Particle Technology 1980, p. 1064, Amsterdam (1980).

5) Naito, M. and G. Jimbo: Funsai (The Micromeritics), No. 29, 104 (1985).

6) Naito, M., N. Kato, G. Jimbo and T. Yokoyama: J. Soc. Powder Technol., Japan, 23, 500 (1986).

7) Naito, M., S. Usuda and G. Jimbo: Kagaku Kogaku Ronbunshu, 12, 495 (1986).

8) Nash, J.H., G.G Leiter and A.P. Johnson: $I \& E C$, Product Research and Development, 4, 140 (1965).

9) Rumpf, H.: Chem. Ing. Tech., 42, 538 (1970). 